\title{
Correlated Atom Accelerometers for Mapping the Earth Gravity Field from Space \\ 10502018
}

Thomas Lévèque (GNES) - 09/10/2018

cnes 


\section{Atom interferometry for space geodesy}

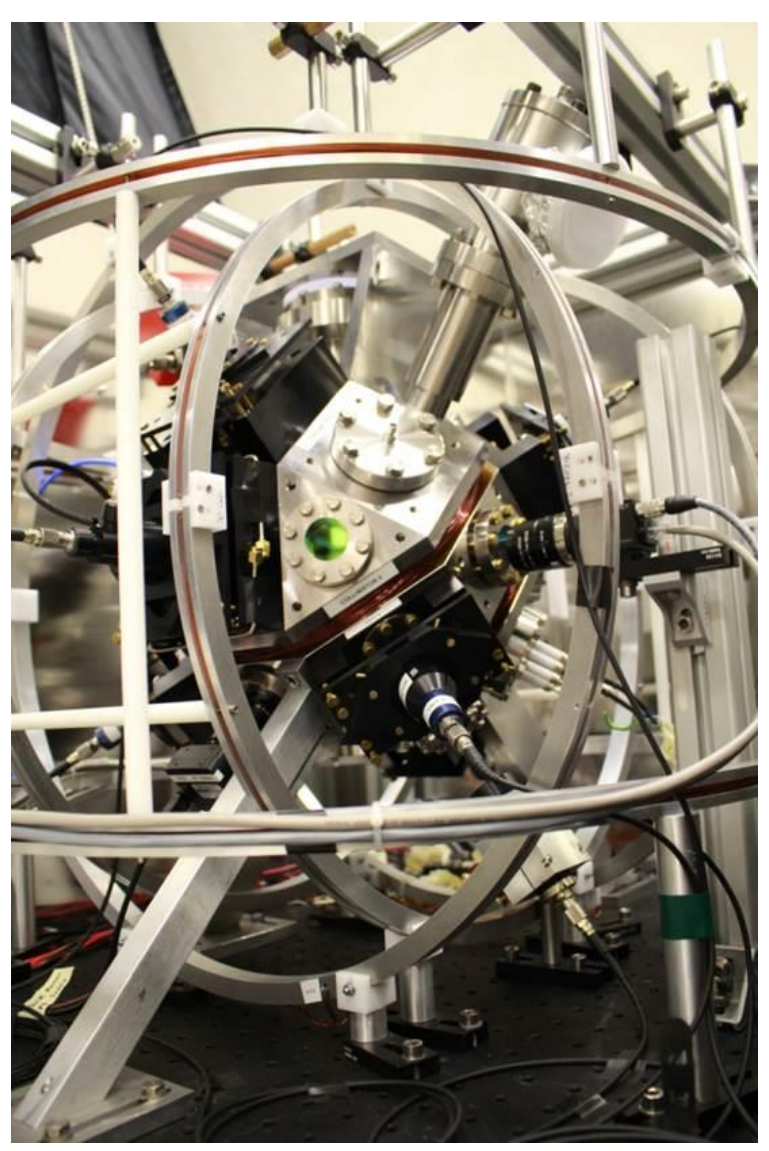

c A disruptive technology:

- Ultra-cold Rb Atoms.

- Matter-Wave Interferometer.

- Cold atom accelerometer

C Space Geodesy:

- Goal: Gravity field mapping from space (GRACE/GOCE).

- Applications:

- Hydrology

- Glaciology

- Oceanography

- Internal Geophysics c Advantages \& Performances:

- Stability

- Accuracy

- Dynamics

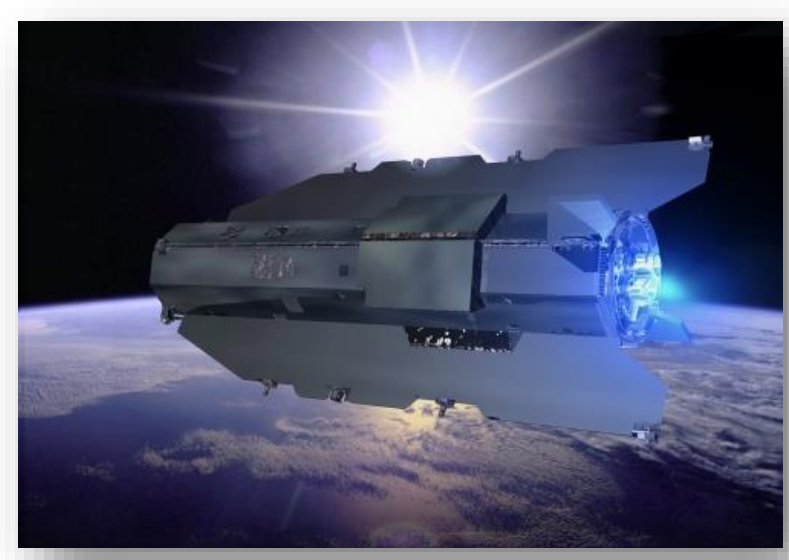




$$
\text { I-General context }
$$

II - Instrument

III - Satellite and mission performances

\author{
IV - Conclusions
}




\section{Summary}

I- General context

II - Instrument

$\bullet$

-

III - Satellite and mission performances

$\bullet$

IV - Conclusions 


\section{Gravity mapping from space}

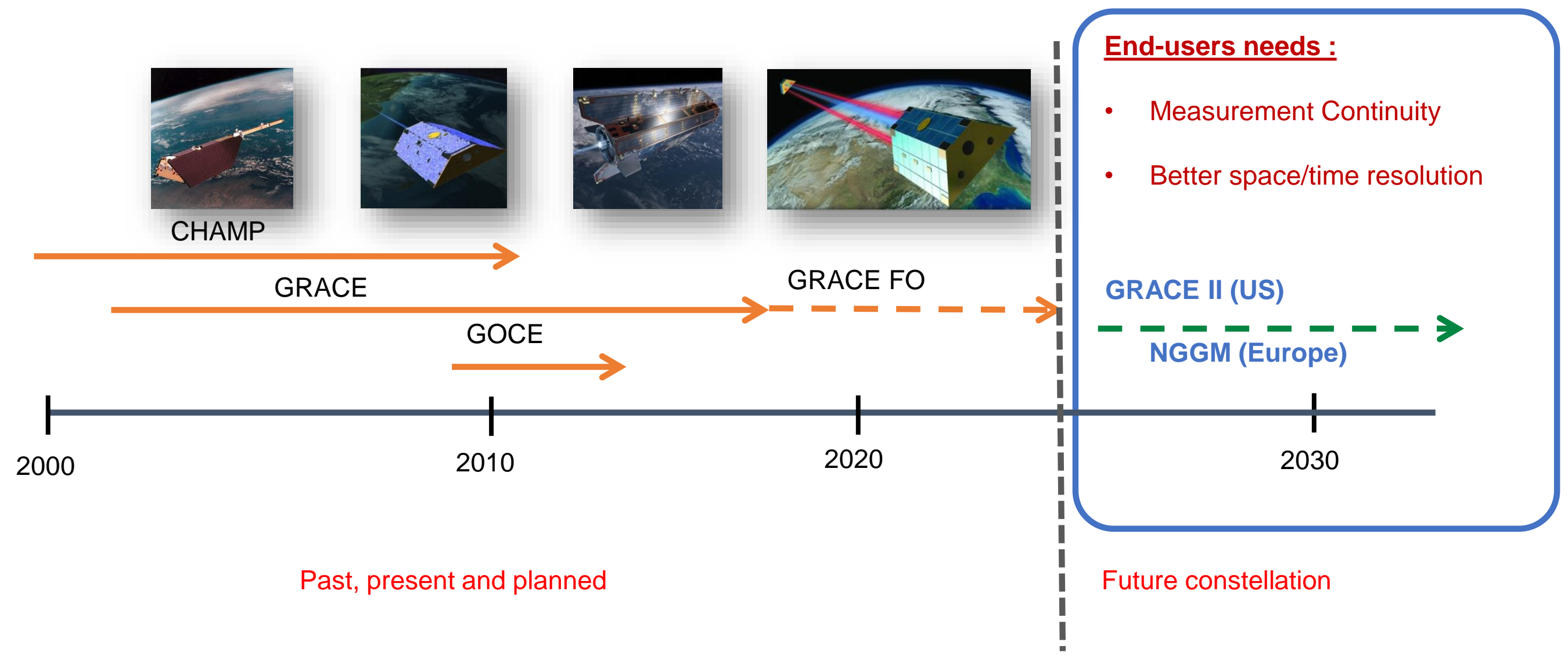




\section{Summary}

\section{| - General context}

II - Instrument

III - Satellite and mission performances

IV - Conclusions 
Atom interferometry \& Gradiometric inertial measurements

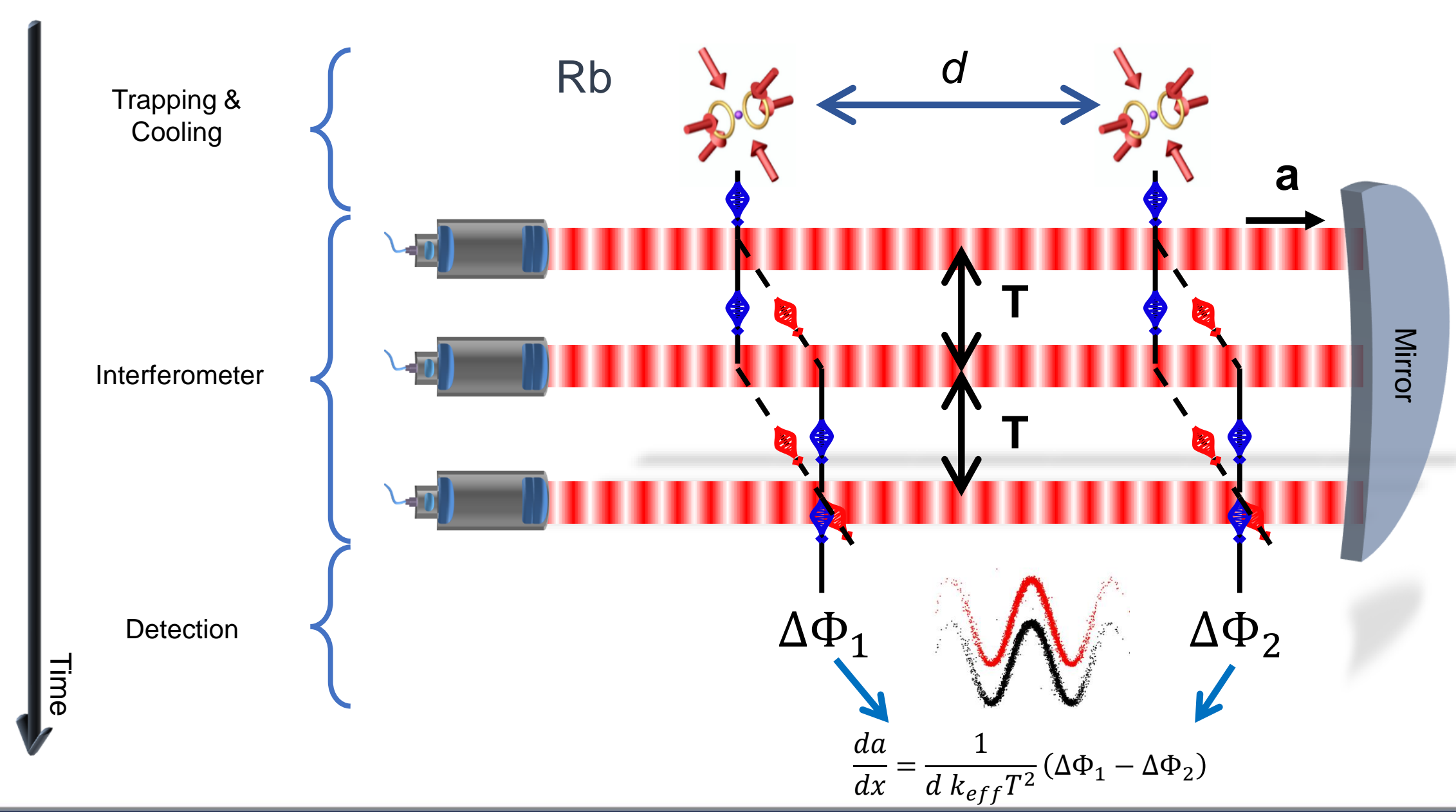




\section{Mission and Instrument Concept}

Satellite 1

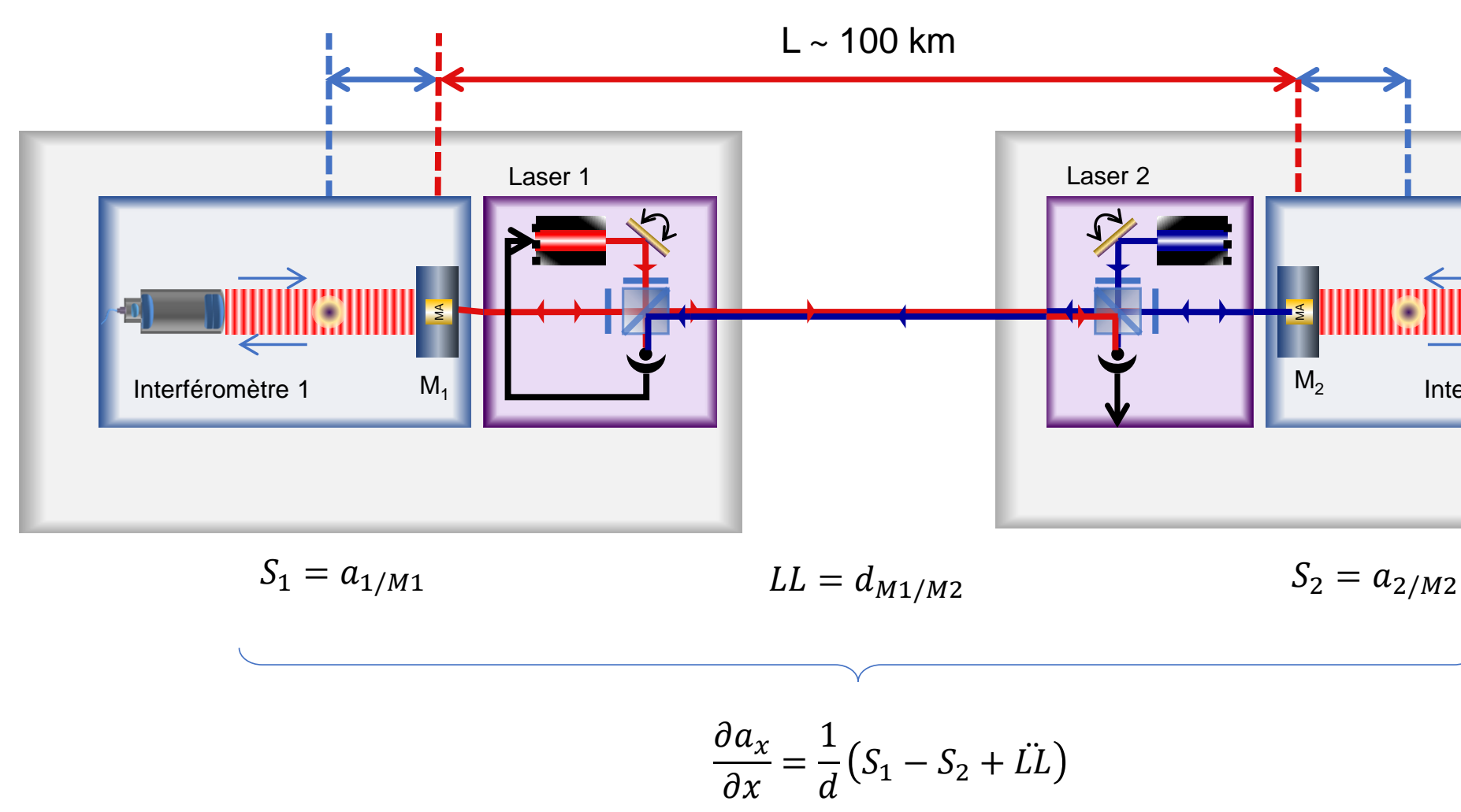

Satellite 2

Sensitivity : $\sim 10 \mu \mathrm{E} . \mathrm{Hz}^{-1 / 2}$ 


\section{Instrument performances}

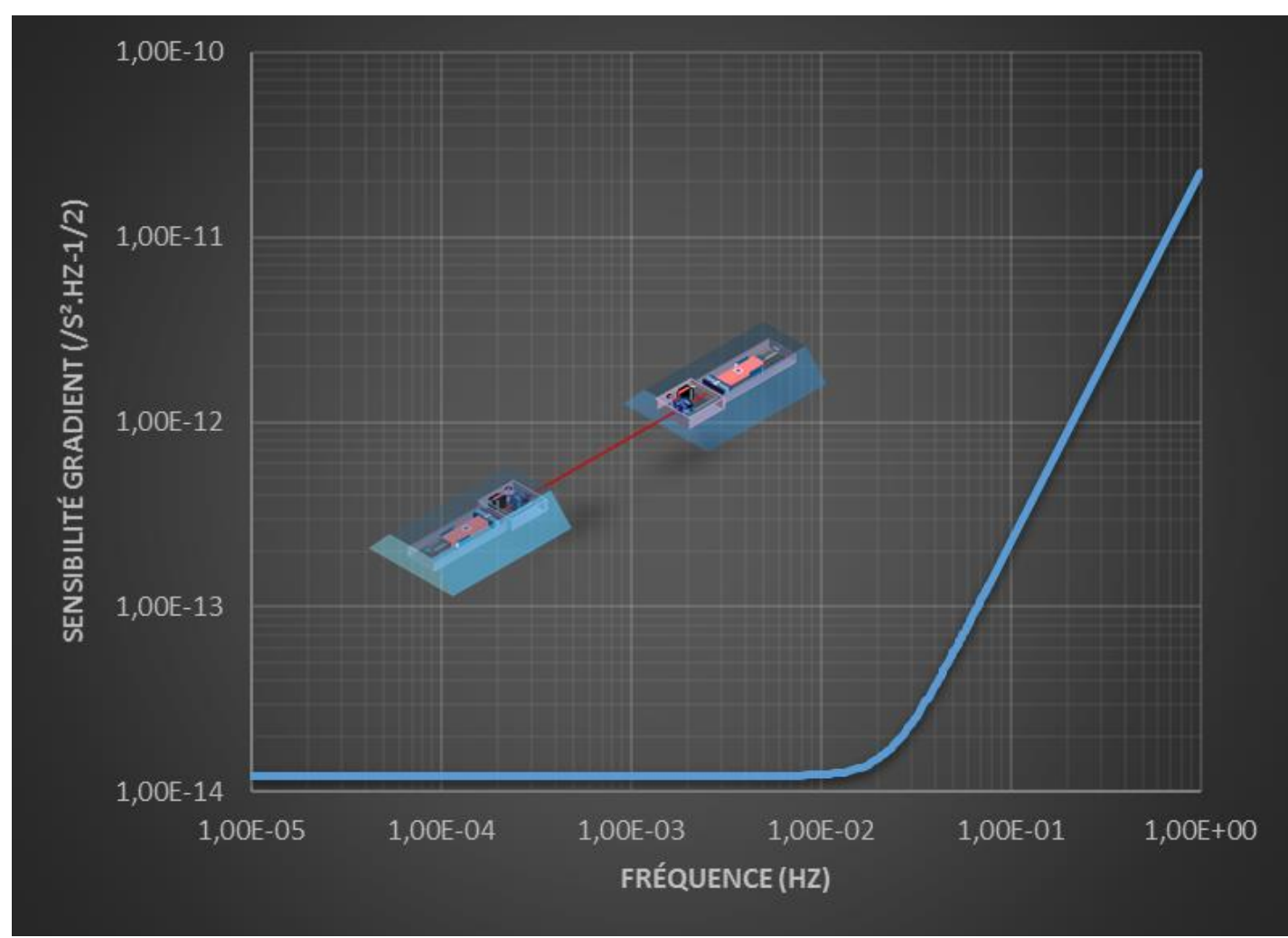


Summary

\section{I - General context}

II - Instrument

-

$\bullet$

III - Satellite and mission performances

IV - Conclusions 


\section{Mission performances simulation}

Signal : hydrology



Synthetic satellite data

Data processing

Variable effects


Instrument noise
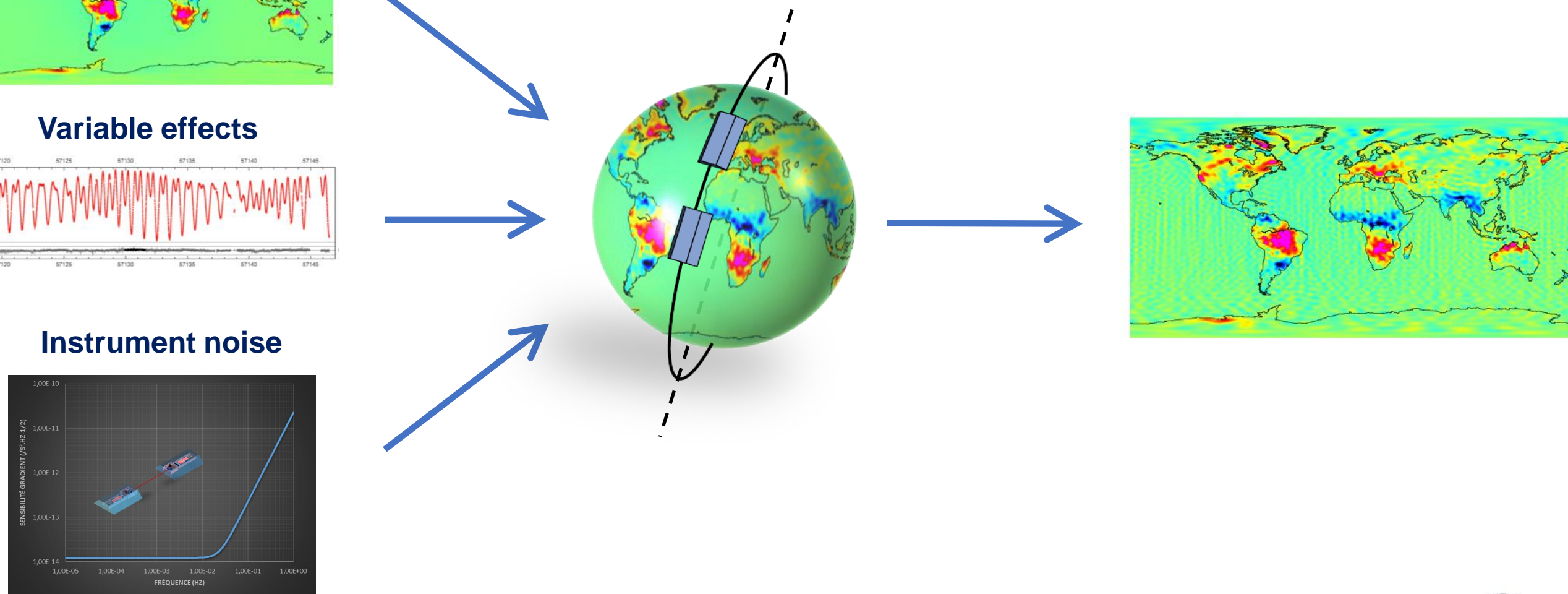


\section{Mission performances simulation}

\section{Input signal}


Signal restitution
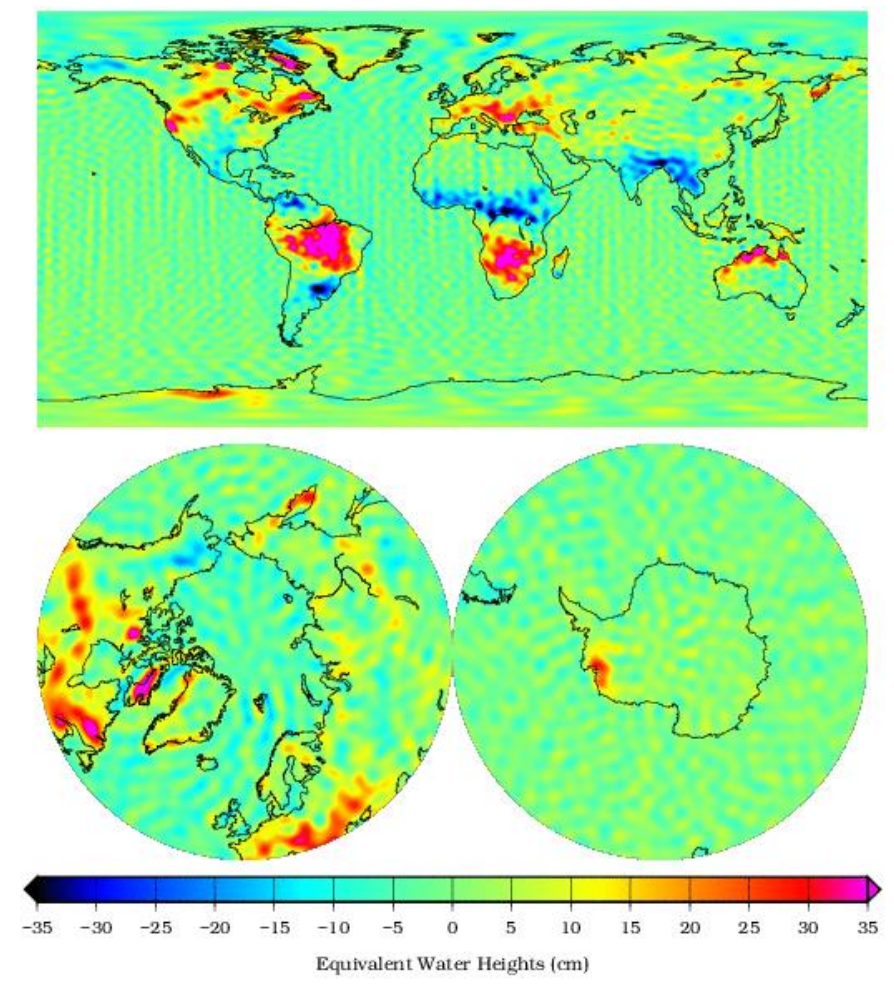


\section{Satellite platform}
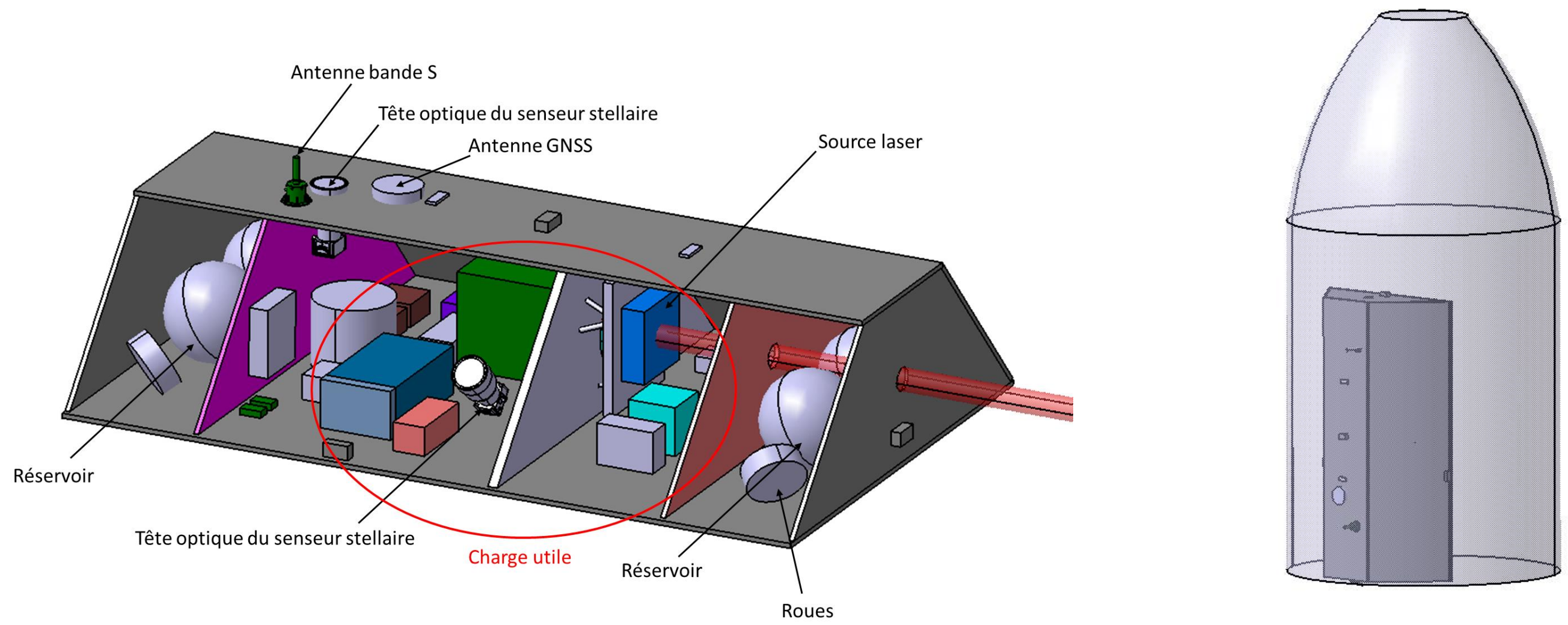


\section{Summary}

I - General context



•

II - Instrument

$\bullet$

$\bullet$

III - Satellite and mission performances

$\bullet$

IV - Conclusions 


\section{Conclusion}

\section{Mission concept:}

Dual satellite mission concept

Composite acceleration gradient measurement

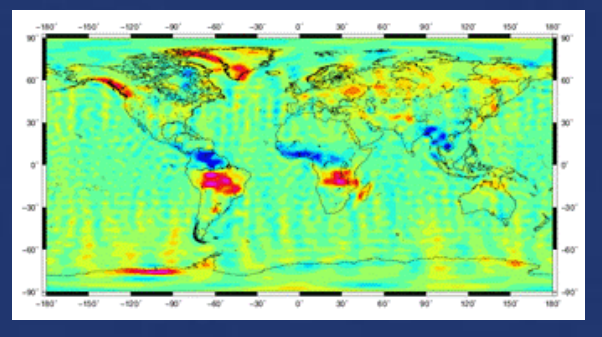

Mission performances:

Numerical simulation of the mission performances

Composite acceleration gradient measurement

\section{Satellite Definition:}

Satellite subsystem definition

Platform study

High TRL subsystem development (Laser source)

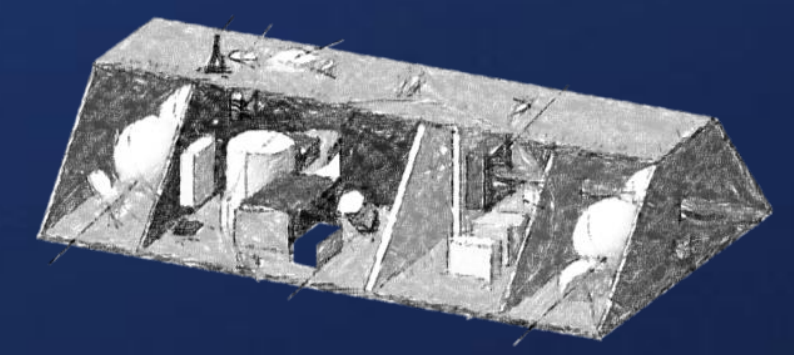




\section{Thank you for your attention}

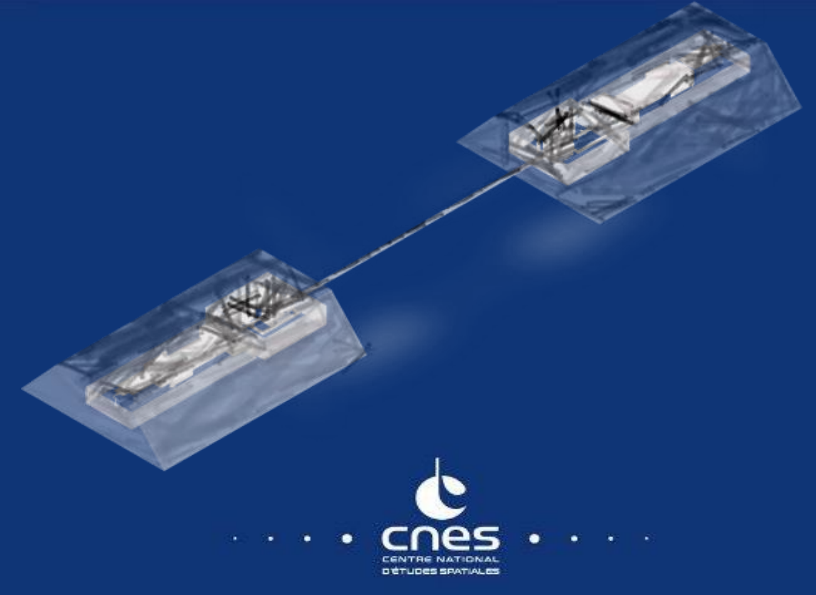




\section{Expected Mission performances}

\begin{tabular}{l|c|rrr}
\multicolumn{1}{c}{ Grandeur } & \multicolumn{1}{c}{ Symbole } & \multicolumn{1}{c}{ Valeur } & \multicolumn{1}{c}{ Unité } \\
\hline Noise LL & $\sigma \mathrm{l}$ & $4,00 \mathrm{E}-08$ & $\mathrm{~m} / \mathrm{Hz} 1 / 2$ \\
\hline Noise Al & $\sigma a$ & $6,13 \mathrm{E}-10$ & $\mathrm{~m} / \mathrm{s}^{2} / \mathrm{Hz} 1 / 2$ \\
\hline Baseline & $\mathrm{L}$ & $1,00 \mathrm{E}+05$ & $\mathrm{~m}$
\end{tabular}
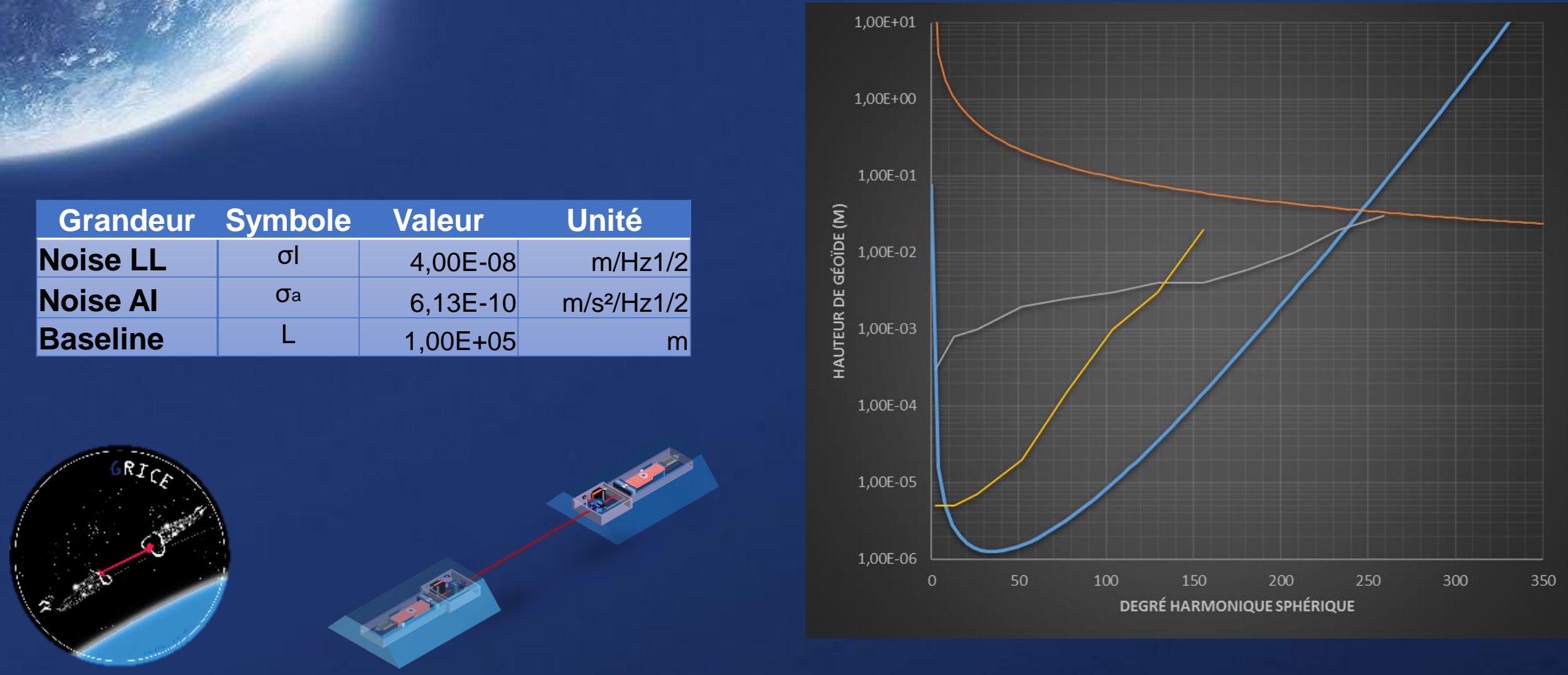

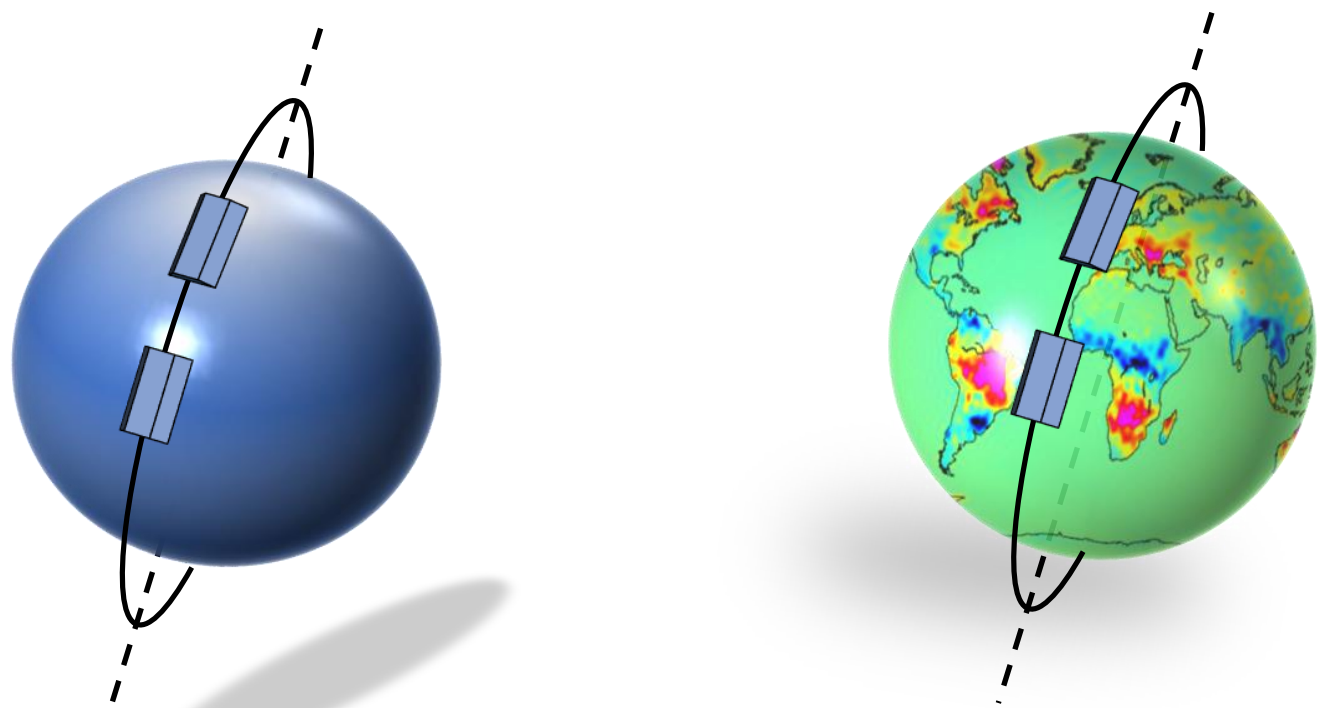